\title{
HIV-indicator-condition-driven HIV testing: clinically effective but still rarely implemented
}

\author{
Authors: Lauren Bull ${ }^{A}$ and Michael Rayment ${ }^{B}$
}

In the UK, outcomes for people living with HIV are excellent. However, a quarter of those living with HIV do not know their status, and almost half are diagnosed late. Strategies to broaden HIV testing are needed. HIV indicator conditions are those thought to be associated with HIV infection because they share risk factors (eg viral hepatitis) or because they arise as a result of early or late immunodeficiency (eg bacterial pneumonia, Kaposi's sarcoma). They comprise all AIDS-defining conditions, but also many non-AIDS-defining conditions spanning the spectrum of medicine. Patients presenting with indicator conditions should routinely be offered an HIV test. This approach is likely to be clinically effective, because knowledge of HIV status is essential in the management of many conditions. It is cost effective if the prevalence of HIV infection is greater than $0.1 \%$. The strategy removes the need for risk assessment, and is acceptable to patients and healthcare practitioners. If broadly implemented, it is likely to be effective at a public health level, and will help to reduce both undiagnosed HIV and late diagnoses of HIV. Here we review the emerging evidence base that supports the value of routine HIV testing in indicator conditions.

\section{Introduction}

In the UK, clinical outcomes for people living with HIV are excellent. 95\% of people living with HIV are accessing specialist care, of whom $88 \%$ are receiving antiretroviral therapy (ART). $86 \%$ of this group has undetectable viral loads - a surrogate marker of treatment success. ${ }^{1}$ However, two features mar the epidemiology of HIV infection in the UK: a persistent proportion of people who do not know they are HIV positive, and a high proportion of people being diagnosed with laterstage HIV infection.

Of the 107,800 people living with HIV in the UK, an estimated $22 \%$ do not know their status. ${ }^{1}$ Knowledge of HIV status is important for public health because it reduces risk behaviour and allows partners to access testing. ${ }^{2}$ Furthermore, knowledge

Authors: ${ }^{\text {A }}$ specialty registrar, Directorate of HIV/GU Medicine, Chelsea and Westminster Hospital NHS Foundation Trust, London, UK; ${ }^{\text {B }}$ consultant, Directorate of HIV/GU Medicine, Chelsea and Westminster Hospital NHS Foundation Trust, London, UK of status is a prerequisite to accessing ART, which has been proven to reduce transmission. ${ }^{3-5} 47 \%$ of infections diagnosed in 2012 were diagnosed late (ie patients have a CD4 cell count of below 350 cells $/ \mu \mathrm{L}$ at the time of diagnosis). The CD4 count at diagnosis is a strong predictor of short-term and long-term morbidity and mortality. Patients whose CD4 counts are below 350 cells/ $\mu \mathrm{L}$ are 10 times more likely to die in the first year after diagnosis than those with CD4 counts greater than 350 cells $/ \mu \mathrm{L} .{ }^{1}$

Thus, reducing the size of the undiagnosed fraction, and the proportion of diagnoses made late, is highly likely to yield significant individual and public health benefits. Timely testing for HIV infection remains the key intervention to tackle both problems.

\section{HIV testing and indicator conditions}

UK guidelines for HIV testing published in 2008 aimed to normalise, destigmatise and expand HIV testing. ${ }^{6}$ They recommended routine screening for all adults accessing primary and secondary care in high-prevalence areas (defined as a local diagnosed prevalence $>0.2 \%$ ), in addition to targeted testing for those in higher-risk groups on the basis of demography and risk factors. Furthermore, the guidelines recommended the routine offer of an HIV test to all patients of unknown HIV status presenting with a number of medical conditions (known as HIV indicator conditions (ICs)) whether or not they belong to a higher-risk group. The National Institute for Health and Care Excellence has since ratified this guidance. ${ }^{7,8}$ An IC-based approach has also been endorsed across Europe. ${ }^{9}$

Since 2009, numerous interventional, prospective studies in the UK have demonstrated the feasibility and high acceptability of delivering routine HIV testing programmes to large, unselected populations in areas with a high prevalence of HIV infection in line with these recommendations. ${ }^{10-12}$ Although acceptable to most staff and patients and clinically effective, a 2014 systematic review and meta-analysis suggests that the major barrier to implementation of the 2008 guidance resides with healthcare workers. ${ }^{13}$

One advantage of IC-guided HIV testing is the removal of the need for HIV risk assessment, as the IC itself acts as trigger for the routine offer of an HIV test, independent of other risk factors. This has the potential to reduce HIV-related stigma and discrimination and increase the number of tests offered and accepted, thus normalising HIV testing. HIV ICs can be 
divided into two categories: conditions that are AIDS defining and those that are not AIDS defining but are associated with an undiagnosed prevalence of HIV infection over $0.1 \%$. They are thought to occur more frequently in people living with HIV, either because they share a common mode of transmission (eg viral hepatitis, syphilis) or because they occur as a result of the characteristic immunodeficiency associated with HIV infection (eg cervical intraepithelial neoplasia). Non-AIDS-defining ICs can present at earlier stages in the natural history of HIV infection, and hence routine HIV testing could facilitate earlier diagnosis. A proposed list of ICs is shown in Table 1.

Diagnosis of concurrent HIV infection in patients presenting with ICs is essential because it can inform choices of treatment (eg antiviral therapy for hepatitis B and C), treatment with effective ART might be necessary for the optimal care of the underlying condition (eg HIV-related thrombocytopenia) and treatment can be complicated by concurrent immunosuppression (eg with chemotherapy).

Additionally, data from the USA and France suggest that delivering testing in settings or populations in which the prevalence of HIV infection is at least $0.1 \%$ is cost effective. ${ }^{14-16}$ Thus, routine screening in conditions associated with such a prevalence is likely to be economically viable. However, a 2010 survey showed poor awareness of HIV IC-testing guidance across medical specialities. ${ }^{17}$ Of 17 medical royal colleges, faculties and professional organisations approached, 11 organisations reported awareness of the guidelines, yet only five had included HIV testing in any of their own clinical guidelines pertaining to the management of individuals presenting for the care of ICs. An audit examining application of HIV testing for ICs in the UK showed poor application of the guidelines with resultant missed opportunities for diagnosis. ${ }^{18} \mathrm{~A}$ review of ten UK studies reporting HIV testing rates in patients presenting with ICs showed that just 22\% (95\% confidence interval 14-31) received an HIV test. ${ }^{13}$ There are few prospectively collected data on HIV test offers for patients presenting with ICs. However, Public Health England collects routine data on

\section{Key points}

Increased HIV testing remains of paramount importance to identify patients who remain undiagnosed.

Indicator-condition-guided testing removes the need for individual risk assessment and normalises HIV testing.

Ascertaining HIV status is likely to inform best management of indicator conditions.

Routine testing for HIV is cost effective at a prevalence level of above $0.1 \%$.

Prevalence of previously undiagnosed HIV has been demonstrated to be more than $0.1 \%$ in a wide variety of indicator conditions and therefore HIV testing should be done routinely.

KEYWORDS: HIV, indicator conditions, HIV testing, public health
HIV testing in tuberculosis clinics. In 2012, $66.8 \%$ of eligible individuals were tested for HIV, climbing to $81.1 \%$ in 2013 , once routine HIV testing was included as a key performance indicator. ${ }^{19}$

Although data exist to describe the occurrence of ICs in patients known already to be living with HIV, a systematic review in 2012 showed few published data for the prevalence of previously undiagnosed HIV infection in individuals of unknown HIV status presenting with ICs. ${ }^{20}$ However, data that support the value and effectiveness of IC-driven HIV testing are now emerging.

A retrospective case-control study (939 cases and 2,576 controls) of primary care attendees in the UK showed that 12 of the 37 non-AIDS-related ICs in Table 1 were significantly associated with subsequent HIV diagnosis. ${ }^{20}$ The strongest associations were bacterial pneumonia (odds ratio 47.7, 95\% confidence interval (CI) 5.6-404.0), oral candidiasis $(29.4,6.9-$ 125.5), and herpes zoster $(25.4,8.4-76.1)$. Signs and symptoms most associated with HIV were weight loss (13.4, 5.0-36.0), pyrexia of unknown origin $(7.2,2.8-18.7)$ and diarrhoea (one or two consultations). However, it is important to note that 697 (74.2\%) of the HIV cases did not present to primary care with any of the listed ICs before their HIV diagnosis. It is assumed that the majority were thus asymptomatic.

The first prospective data for the prevalence of HIV infection with certain indicator conditions were provided by the HIV Indicator Disease Across Europe (HIDES 1) study. ${ }^{22}$ During this pilot study, HIV tests were routinely offered to patients of unknown HIV status presenting with eight ICs (sexually transmitted infection, lymphoma, cervical or anal cancer or dysplasia, herpes zoster, hepatitis B or C virus infection, mononucleosis-like illness, unexplained leucocytopenia or thrombocytopenia, and seborrhoeic dermatitis) at 17 centres in 14 countries across Europe. The primary outcome was a previously undiagnosed prevalence of HIV infection of greater than $0.1 \%$ (the threshold for cost-effectiveness). Of the 3,588 people tested, 66 received a newly diagnosis with HIV, equating to an overall prevalence of previously undiagnosed HIV of $1.8 \%$ (95\% CI 1.42-2.34). All eight ICs individually fulfilled the study's criterion of demonstrating an HIV prevalence of over $0.1 \%$.

Of the patients who tested HIV positive, 13 (19.7\%) had experienced potential HIV-related symptoms, and 7 (10.6\%) individuals had been admitted to hospital, most with a potential AIDS diagnosis or infection. 34 (52\%) of the newly diagnosed individuals had previously tested negative (median time since last test 1.58 years), which, together with the high median CD4 count at diagnosis ( 400 cells/ $\mu \mathrm{L})$ adds weight to this strategy being effective in diagnosing HIV at an early stage in its natural history. When controlling for recognised demographic and behavioural risk factors for HIV infection, the prevalence in the IC population was still high. Staff involved in the study identified barriers to delivering IC-guided testing, including operational issues, attitudinal issues, and training needs. However, in most settings these problems were not reflected in the high offer and uptake rates.

In a follow-on study, HIDES 2, both the number of patients recruited and the ICs examined were expanded (Table 2$){ }^{23}$ In total, 9,471 participants were recruited, 235 of whom tested positive for HIV; thus, the prevalence of HIV infection was 


\section{Table 1. Clinical indicator conditions for adult HIV infection.}

\section{AIDS-defining conditions Other conditions in which HIV testing should be offered}

\begin{tabular}{|c|c|c|}
\hline Respiratory & Tuberculosis pneumocystis & Bacterial pneumonia aspergillosis \\
\hline \multirow[t]{8}{*}{ Neurology } & Cerebral toxoplasmosis & Aseptic meningitis or encephalitis \\
\hline & Primary cerebral lymphoma & Cerebral abscess \\
\hline & Cryptococcal meningitis & Space occupying lesion of unknown cause \\
\hline & Progressive multifocal & Guillain-Barré syndrome \\
\hline & leucoencephalopathy & Transverse myelitis \\
\hline & & Peripheral neuropathy \\
\hline & & Dementia \\
\hline & & Leucoencephalopathy \\
\hline \multirow[t]{3}{*}{ Dermatology } & Kaposi's sarcoma & Severe or recalcitrant seborrhoeic dermatitis \\
\hline & & Severe or recalcitrant psoriasis \\
\hline & & Multidermatomal or recurrent herpes zoster \\
\hline \multirow[t]{7}{*}{ Gastroenterology } & Persistent cryptosporidiosis & Oral candidiasis \\
\hline & & Oral hairy leukoplakia \\
\hline & & Chronic diarrhoea of unknown cause \\
\hline & & Weight loss of unknown cause \\
\hline & & Salmonella, Shigella or Campylobacter enteritis \\
\hline & & Hepatitis B infection \\
\hline & & Hepatitis C infection \\
\hline \multirow[t]{6}{*}{ Oncology } & Non-Hodgkin lymphoma & Anal cancer or anal intraepithelial dysplasia \\
\hline & & Lung cancer \\
\hline & & Seminoma \\
\hline & & Head and neck cancer \\
\hline & & Hodgkin lymphoma \\
\hline & & Castleman's disease \\
\hline \multirow[t]{2}{*}{ Gynaecology } & Cervical cancer & Vaginal intraepithelial neoplasia \\
\hline & & Cervical intraepithelial neoplasia (grade II or above) \\
\hline Haematology & & $\begin{array}{l}\text { Any unexplained blood dyscrasia including thrombocytopenia, neutropenia and } \\
\text { lymphopenia }\end{array}$ \\
\hline \multirow[t]{2}{*}{ Ophthalmology } & Cytomegalovirus retinitis & Infective retinal diseases including herpes viruses and toxoplasma \\
\hline & & Any unexplained retinopathy \\
\hline \multirow{3}{*}{$\begin{array}{l}\text { Ear, nose and } \\
\text { throat }\end{array}$} & - & Lymphadenopathy of unknown cause \\
\hline & & Chronic parotitis \\
\hline & & Lymphoepithelial parotid cysts \\
\hline \multirow[t]{4}{*}{ Other } & - & Mononucleosis-like syndrome (primary HIV infection) \\
\hline & & Pyrexia of unknown origin \\
\hline & & Any lymphadenopathy of unknown cause \\
\hline & & Any sexually transmitted infection \\
\hline
\end{tabular}

2.5\% (95\% CI 2.2-2.8). Cost-effectiveness was established for 10 of 14 ICs, in which a prevalence of $>0.1 \%$ was definitively demonstrated. For the remaining conditions, relatively few patients were tested and there were few events.

These prospective studies have definitively shown the yield and value of routine HIV testing in patients presenting with the indicator conditions studied. All conditions associated with a proven prevalence of HIV infection of more than $0.1 \%$ should be adopted into HIV testing and IC specialty guidelines on both national and European levels. Further studies should be implemented to assess routine HIV testing in other putative ICs. Staff training must be incorporated to overcome barriers at the provider level, and pathways to HIV specialist care developed. Where data are available, the acceptability of HIV 


\begin{tabular}{|c|c|c|c|c|}
\hline & Tested, $\mathbf{n}$ & HIV positive, $n$ & Prevalence, \% & $95 \%$ confidence interval \\
\hline Lymphoma & 588 & 4 & 0.7 & $0.6-1.3$ \\
\hline CIN2/3/cervical cancer & 1,339 & 13 & 1.0 & $0.5-1.5$ \\
\hline Anal dysplasia or cancer & 53 & 0 & 0 & $\mathrm{n} / \mathrm{a}$ \\
\hline Hepatitis B & 1,126 & 13 & 1.2 & $0.5-1.8$ \\
\hline Hepatitis C & 1,751 & 41 & 2.3 & $1.6-3.1$ \\
\hline Hepatitis B and C & 73 & 7 & 9.6 & $2.8-16.3$ \\
\hline Mononucleosis-like illness & 734 & 39 & 5.3 & $3.7-6.9$ \\
\hline Leucocytopenia/thromobocytopenia & 401 & 16 & 4.0 & $2.1-5.9$ \\
\hline Seborrhoeic dermatitis & 299 & 6 & 2.0 & $0.4-3.6$ \\
\hline Pneumonia & 1,881 & 61 & 3.2 & $2.4-4.0$ \\
\hline Lymphadenopathy & 722 & 32 & 4.4 & $2.9-5.9$ \\
\hline Peripheral neuropathy & 84 & 2 & 2.4 & $0.0-5.1$ \\
\hline Lung cancer & 144 & 0 & 0 & $\mathrm{n} / \mathrm{a}$ \\
\hline Severe psoriasis & 276 & 1 & 0.4 & $0.0-1.1$ \\
\hline TOTAL & 9,471 & 235 & 2.5 & $2.2-2.8$ \\
\hline
\end{tabular}

CIN = cervical intraepithelial neoplasia.

testing to patients presenting with ICs is high, with the vast majority accepting the offer of a test.

Testing for HIV infection in the era of effective antiretroviral therapy should be simple, and within the remit of any healthcare worker. ${ }^{6,24}$ There is no requirement for lengthy pre-test counselling. Consenting for an HIV test should involve the same discussion points as the consenting process for any diagnostic test - namely, a discussion of the risks and benefits of the test, and an explanation of the different results it could yield. This information may be given verbally, or in writing (eg via a patient information leaflet). It is always in a patient's interests to know their HIV status, be it negative or positive. Most patients accept the offer of a test, but if a patient declines a test, their reasons for declining should be probed, and any misinformation corrected. For example, having an HIV test in itself has no impact on insurance applications - a common misconception. Arrangements should be made for the patient to access their result in a timely manner. Pathways for timely referral into specialist care in the event of a positive result should be developed as required.

\section{Conclusion}

Indicator disease testing has been proven to effective. It helps clinicians to focus on the clinical syndrome without having to ask questions about risk behaviour. Ascertaining HIV status is likely to be important for the management of the IC. Adoption of IC-driven testing could help to identify earlier-stage HIV infection, and thus result in individual and public health benefits. Cost-effectiveness has now been proven in several ICs associated with a prevalence of HIV infection greater than $0.1 \%$. We would strongly recommend that clinicians offer a routine HIV test to any patient presenting with these conditions. However, closing the translational gap remains a challenge. We would argue that all clinicians providing care to individuals with HIV ICs critically appraise their HIV testing practice and take steps to adopt routine HIV testing for all.

\section{References}

1 Health Protection Agency. HIV in the UK, 2014. London: Public Health England, 2014.

2 Marks G, Crepaz N, Janssen RS. Estimating sexual transmission of HIV from persons aware and unaware that they are infected with the virus in the USA. AIDS 2006;20:1447-50.

3 Reynolds SJ, Makumbi F, Nakigozi G et al. HIV-1 transmission among HIV-1 discordant couples before and after the introduction of antiretroviral therapy. AIDS 2011;25:473-4.

4 Donnell D, Baeten JM, Kiarie J et al. Heterosexual HIV-1 transmission after initiation of antiretroviral therapy: a prospective cohort analysis. Lancet 2010;375:2092-8.

5 Cohen MS, Smith MK, Muessig KE et al. Antiretroviral treatment of HIV-1 prevents transmission of HIV-1: where do we go from here? Lancet 2013;382:1515-24.

6 British HIV Association, British Association for Sexual Health and HIV, British Infection Society. UK national guidelines for HIV testing 2008. London, British HIV Association, 2008.

7 National Institute for Health and Care Excellence. Increasing the uptake of HIV testing among men who have sex with men. London: National Institute for Health and Care Excellence, 2011.

8 National Institute for Health and Care Excellence. Increasing the uptake of HIV testing among black Africans in England. London: National Institute for Health and Clinical Excellence, 2011.

9 Gazzard B, Clumeck N, D’Arminio Monforte A, Lundgren JD. Indicator diseases-guided testing for HIV - the next step for Europe? HIV Med 2008;9(suppl 2):34-40.

10 Rayment M, Thornton A, Mandalia S et al. HIV testing in nontraditional settings - the HINTS study: a multi-centre observational study of feasibility and acceptability. PLoS One 2012;7:e39530.

11 Palfreeman A, Nyatsanza F, Farn H et al. HIV testing for acute medical admissions: evaluation of a pilot study in Leicester, England. Sex Transm Infect 2013;89:308-10. 
12 Ellis S, Graham L, Price DA, Ong ELC. Offering HIV testing in an acute medical admissions unit in Newcastle upon Tyne. Clin Med 2011;11:541-3.

13 Elmahdi R, Gerver SM, Gomez Guillen G et al. Low levels of HIV test coverage in clinical settings in the UK: a systematic review of adherence to 2008 guidelines. Sex Transm Infect 2014;90:119-24.

14 Paltiel AD, Weinstein MC, Kimmel AD et al. Expanded screening for HIV in the United States - an analysis of cost-effectiveness. N Engl J Med 2005;352:586-95.

15 Sanders GD, Bayoumi AM, Sundaram V et al. Cost-effectiveness of screening for HIV in the era of highly active antiretroviral therapy. N Engl J Med 2005;352:570-85.

16 Yazdanpanah Y, Sloan CE, Charlois-Ou C et al. Routine HIV screening in France: clinical impact and cost-effectiveness. PLoS One 2012;15:e13132.

17 Health Protection Agency. Time to test for HIV: expanding HIV testing in healthcare and community services in England. London: Health Protection Agency, 2011.

18 Rayment M, Rae C, Jakobsen M et al. High yield, but variable coverage of HIV indicator conditions across the UK. HIV Med 2014;15(suppl 3):17-159.

19 Public Health England. Collaborative tuberculosis strategy for England. London: Public Health England, 2014.
20 HIV in Europe Secretariat. HIV indicator conditions: guidance for implementing hiv testing in adults in health care settings. Brussels: HIV in Europe, 2013.

21 Damery S, Nichols L, Holder R et al. Assessing the predictive value of HIV indicator conditions in general practice: a case-control study using the THIN database. Br J Gen Pract 2013;63:e370-7.

22 Sullivan A. Raben D, Reekie J et al. Feasibility and effectiveness of indicator condition-guided testing for HIV: results from HIDES I (HIV Indicator Diseases Across Europe Study). PLoS One 2013;8:e52845.

23 Rayment M, Kutsyna G, Mocroft A et al. The effectiveness of indicator disease-based HIV testing across Europe - results from a prospective multi-centre study. HIV Med 2015; 16(suppl 2):1-11.

24 Rayment M, Asboe D, Sullivan AK. HIV testing and management of newly diagnosed HIV. BMJ 2014;349:g4275.

Address for correspondence: Dr M Rayment, Chelsea and Westminster Hospital NHS Foundation Trust, 369 Fulham Road, London SW10 9NH, UK.

Email: michaelrayment@nhs.net 